\title{
Nonlinear Hyperbolic Smoothing at a Focal Point
}

\author{
JeAn-LuC Joly, Guy Metivier, \\ \& JEFFREY RAUCH
}

\section{Introduction}

We construct real-valued finite energy solutions of the dissipative nonlinear wave equation

$$
\square u+\left|u_{t}\right|^{h-1} u_{t}=0, \quad \square:=\partial_{t}^{2}-\Delta_{x}, \quad 1<h \in \mathbb{R},
$$

which have singularities that are partially smoothed after a focus. Here $\{t, x\} \in$ $\mathbb{R}^{1+d}$, with spatial dimension $d \geq 2$.

A striking classical result of Lions and Strauss [LS] shows that (1.1) is a wellbehaved evolution equation in $t \geq 0$ in all dimensions. Two underlying estimates are used in establishing this result. The first is that solutions have nonincreasing energy. With

$$
E(u, t):=\int_{\mathbb{R}^{d}} \frac{u_{t}^{2}}{2}+\frac{\left|\nabla_{x} u\right|^{2}}{2} d x,
$$

one has

$$
E(u, t)=E(u, 0)-\int_{0}^{T} \int_{\mathbb{R}^{d}} \frac{\left|u_{t}\right|^{h+1}}{h+1} d x d t \leq E(u, 0) .
$$

More generally, one has a contractivity estimate that relies on the monotonicity of the nonlinear function

$$
F_{h}(s):=\left|s^{h-1}\right| s .
$$

Precisely,

$$
\begin{aligned}
E(u-v, t) & =E(u-v, 0)-\int_{0}^{T} \int_{\mathbb{R}^{d}}\left(u_{t}-v_{t}\right)\left(F_{h}\left(u_{t}\right)-F_{h}\left(v_{t}\right)\right) d x d t \\
& \leq E(u-v, 0) .
\end{aligned}
$$

The energy dissipation identity is the case $v=0$ of the contractivity identity. These estimates lead to the following fundamental results of Lions and Strauss.

THEOREM 1.1 [LS]. If $\{f, g\} \in H^{1}\left(\mathbb{R}^{d}\right) \times L^{2}\left(\mathbb{R}^{d}\right)$ then there is a unique solution $u$ to (1.1) with

Received June 16, 1999. Revision received February 25, 2000.

Research partially supported by the U.S. National Science Foundation, U.S. Office of Naval Research, and the NSF-CNRS cooperation program under grant numbers NSF-DMS-9203413, NSFDMS-9803296, OD-G-N0014-92-J-1245, and NSF-INT-9314095, respectively, and by the CNRS through the Groupe de Recherche G1180 POAN. 


$$
u \in C\left(\left[0, \infty\left[; H^{1}\left(\mathbb{R}^{d}\right)\right), \quad u_{t} \in C\left(\left[0, \infty\left[; L^{2}\left(\mathbb{R}^{d}\right)\right) \cap L^{h+1}\left(\left[0, \infty\left[\times \mathbb{R}^{d}\right),\right.\right.\right.\right.\right.\right.
$$

with Cauchy data

$$
\left.u\right|_{t=0}=f,\left.\quad u_{t}\right|_{t=0}=g .
$$

In addition, the energy laws (1.3) and (1.4) are satisfied by pairs of such solutions as well as by the local versions in the truncated cones $\Gamma(\underline{x}, R, T):=\{|x-\underline{x}|<$ $R-t, 0<t<T \leq R\}$.

The energy law in $\Gamma(\underline{x}, R, R)$ shows that two solutions whose Cauchy data agree on $|x-\underline{x}| \leq R$ must also agree on cone $|x-\underline{x}| \leq R-t$.

Regularity results follow from this by applying the contractivity estimate (1.4) to the solutions $u(t, x)$ and $v=u(t, x+\xi)$. The $H^{1}$ modulus of continuity is defined by

$$
\begin{aligned}
\omega(u, t, h)^{2}:= & \sup _{0<|\xi| \leq h}\left\|\nabla_{x} u(t, x)-\nabla_{x} u(t, x+\xi)\right\|_{L^{2}\left(\mathbb{R}^{d}\right)}^{2} \\
& +\left\|u_{t}(t, x)-u_{t}(t, x+\xi)\right\|_{L^{2}\left(\mathbb{R}^{d}\right)}^{2} .
\end{aligned}
$$

COROLlaRY 1.2 [LS]. If $u$ is one of the solutions from Theorem 1 , then the $H^{1}$ modulus of continuity $\omega(u, t, h)$ is a decreasing function of $t$. It follows that, if $f, g \in H^{\sigma+1} \times H^{\sigma}$ with $\left.\left.\sigma \in\right] 0,1\right]$, then

$$
u \in L^{\infty}\left(\left[0 , \infty [ ; H ^ { \sigma + 1 } ( \mathbb { R } ^ { d } ) ) \text { and } u _ { t } \in L ^ { \infty } \left(\left[0, \infty\left[; H^{\sigma}\left(\mathbb{R}^{d}\right)\right) .\right.\right.\right.\right.
$$

For $\sigma \in] 0,1[$ one has continuity in time; that is,

$$
u \in C\left(\left[0 , \infty [ ; H ^ { \sigma + 1 } ( \mathbb { R } ^ { d } ) ) \text { and } u _ { t } \in C \left(\left[0, \infty\left[; H^{\sigma}\left(\mathbb{R}^{d}\right)\right) .\right.\right.\right.\right.
$$

This shows that $H^{s}$ regularity for $1 \leq s \leq 2$ propagates forward in time.

The major interest of these results is that they define a strongly nonlinear evolution. By any known measure, these problems are supercritical when $d$ is large. These problems can not be attacked by using the basic estimates and then treating the nonlinear term as a perturbation, writing $u=-\square^{-1}\left(u_{t}^{h}\right)$. In particular, for $d$ large and $h \in \mathbb{Z}_{\text {odd }}$, the nonlinearity is polynomial and it is not known whether the solutions with data in $C_{0}^{\infty}$ are $C^{\infty}$. Equivalently, it is not known if such solutions are locally Lipshitzean.

Our main result is the construction of compactly supported solutions that are smoother in $\{t \geq 1\}$ than they are in $\{0 \leq t<1\}$ (see Figure 1). This includes an explicit solution in closed form computed in Section 5 .

The examples cannot be locally Lipshitzean because the result of [GR] shows that if a solution has $\nabla_{t, x} u \in L_{\text {loc }}^{\infty}$ then its $H_{\text {loc }}^{s}$ regularity does not change with time. In particular, in the 1-dimensional case, if the Cauchy data satisfies $\nabla_{t, x} u(0, x) \in$ $L_{\mathrm{loc}}^{\infty}$ then the solution is Lipshitzean and hence the $H^{s}$ regularity is independent of $t \geq 0$. This Lipshitz bound is proved by an argument needed later, so we recall the estimates. Introduce the characteristic combinations

$$
u_{ \pm}:=\partial_{\mp} u:=\left(\partial_{t} \mp \partial_{x}\right) u .
$$

When $d=1$, the differential equation (1.1) takes the characteristic form 


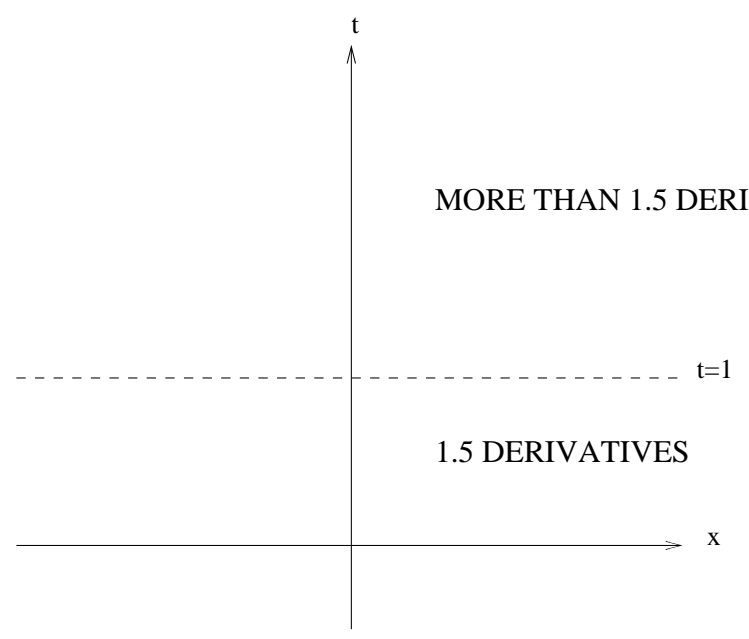

Figure 1 Rough regularity

$$
\left(\partial_{t} \pm \partial_{x}\right) u_{ \pm}+\frac{F_{h}\left(u_{+}+u_{-}\right)}{2^{h}}=0 .
$$

Multiplying by $p u_{ \pm}^{p-1}$ with even integer $p$, adding and then integrating $d x$ shows that

$$
\partial_{t} \int_{\mathbb{R}} u_{+}^{p}+u_{-}^{p} d x=-\frac{p}{2^{h}} \int_{\mathbb{R}}\left(u_{+}^{p-1}+u_{-}^{p-1}\right) F_{h}\left(u_{+}+u_{-}\right) d x .
$$

Since for abitrary real $a, b$ one has $\left(a^{p-1}+b^{p-1}\right) F_{h}(a+b) \geq 0$, it follows that $\int u_{+}^{p}+u_{-}^{p} d x$ is a nonincreasing function of $t$. Passing to the limit $p \rightarrow \infty$ shows that $\sup _{\mathbb{R}} \max \left\{\left|u_{+}\right|,\left|u_{-}\right|\right\}$is a nonincreasing function of $t$. Thus, if $\nabla_{t, x} u(0, \cdot)$ is initially $L^{\infty}$ then it remains so in $t \geq 0$.

Assumption 1.3. Suppose that the initial data $f, g$ are piecewise $C^{2}$, radial, compactly supported, vanish for $|x| \leq 1$, and have singularities only on $|x|=1$. In addition, $f$ is assumed to be continuous and $g+\partial_{r} f$ is not continuous.

When this assumption is satisfied, $\partial_{r} f$ and $g$ are radial piecewise smooth and the locus of singularities is $r=1$. Since $g+\partial_{r} f$ is not continuous, at least one of $g$ and $\partial_{r} f$ must jump at $r=1$. This implies that

$$
\{f, g\} \in H^{\sigma}\left(\mathbb{R}^{d}\right) \times H^{\sigma-1}\left(\mathbb{R}^{d}\right) \Longleftrightarrow \sigma<3 / 2 .
$$

Assumption 1.4. In addition to Assumption 1.3, suppose that $\left(\partial_{t}-\partial_{r}\right) u(0, r)=$ $g-\partial_{r} f$ is continuous at $r=1$.

Since $g-\partial_{r} f$ does not jump, it follows that the jumps of $g$ and $\partial_{r} f$ are equal and nonzero. Assumption 1.4 ensures that a jump discontinuity in $\nabla u$ propagates along the focusing cone $\{|x|=1-t\}$ and that the first derivatives are continuous across the outgoing cone. See Figure 2. 


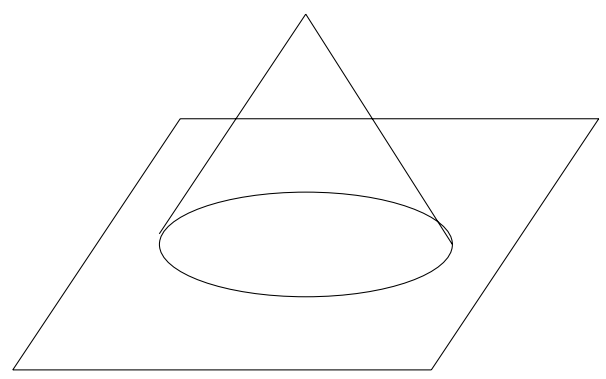

Figure 2 The main singularity for $0<t<1$ is a focusing spherical front

MAIN THEOREM 1.5. Assume that Assumptions 1.3 and 1.4 are satisfied and that $u$ is the solution from Theorem 1.1. Then

$$
u, u_{t} \in L^{\infty}\left(\left[0,1\left[; H^{\sigma}\left(\mathbb{R}^{d}\right) \times H^{\sigma-1}\left(\mathbb{R}^{d}\right)\right) \Longleftrightarrow \sigma<3 / 2\right.\right.
$$

and $u$ is more regular for $t \geq 1$ in the following senses.

(i) If $d>2 h /(h-1)$, then

$$
u \in L^{\infty}\left(\left[1 , \infty [ ; H ^ { 2 } ( \mathbb { R } ^ { d } ) ) \text { and } u _ { t } \in L ^ { \infty } \left(\left[1, \infty\left[; H^{1}\left(\mathbb{R}^{d}\right)\right) .\right.\right.\right.\right.
$$

(ii) If $2 h /(h-1)-1<d \leq 2 h /(h-1)$, let $\alpha:=(2 h /(h-1)-d) / 2 \in[0,1 / 2[$. Then, for all $\varepsilon>0$,

$$
u \in C\left(\left[1, \infty\left[; H^{2-\alpha-\varepsilon}\left(\mathbb{R}^{d}\right)\right) \cap C^{1}\left(\left[1, \infty\left[; H^{1-\alpha-\varepsilon}\left(\mathbb{R}^{d}\right)\right) .\right.\right.\right.\right.
$$

REMARKs. (1) For $h$ fixed, the regularity of the solution for $t \geq 1$ increases linearly from $H^{3 / 2}$ to $H^{2}$ as the dimension increases from $d_{1}(h):=2 h /(h-1)-1$ to $d_{2}(h)=2 h /(h-1)$. For dimensions higher than $d_{2}(h)$, the wave is $H^{2}$ in $t \geq 1$.

(2) Theorem 1 of [GR] shows that, in order for this smoothing to take place, the solutions must not be Lipshitzean. For any $\underline{t} \in] 0,1[$, the solution is uniformly Lipshitzean on $[0, t] \times \mathbb{R}^{d}$, but the sup norm of the derivatives diverges to infinity as $\underline{t} \rightarrow 1$.

(3) What is happening is that an incoming spherical wave focuses at $t=1, x=$ 0 . Approaching the focus, the amplitudes of $u_{t}$ and $u_{t}^{h}$ diverge to infinity. The nonlinear term acts in a dissipative manner. For $d>2 h /(h-1)-1$, the nonlinearity is sufficiently large that the effect of the dissipation is so strong that the solution grows more slowly than it would have in the linear case. The idea of the proof in case (i) is to use the classical energy estimate for the second derivates of $u$ in the domain outside the incoming light cone, that is, $\{\{t, x\}:|x| \geq 1-t\}$. The energy identity involves a boundary term on the incoming light cone $|x|=1-t$. This term is finite for the nonlinear problem and would have been infinite for the linear problem. In this way one shows that the second derivatives at time $t=1$ are square integrable. In case (ii), one shows that they are square integrable with weight $r^{\alpha}, 1>\alpha \geq 0$. Then an inequality of Hardy type finishes the proof. 
(4) There are at least two other circumstances where supercritical damping for the same family of equations has been shown to have a regularizing effect on solutions. The first involves families of oscillatory solutions $u^{\varepsilon}$ whose angular derivatives $\partial_{\omega} \nabla_{t, x} u^{\varepsilon}$ are uniformly bounded in $L^{2}$ at the same time as $\nabla_{t, x} u^{\varepsilon}$ is bounded in $L^{2}$ [JMR2; JMR3; JMR5]. If the initial data is supported in $|x|<1$, is not compact in energy, and has prinicipal oscillations that initially move toward the origin in the sense that $\left(\partial_{t}-\partial_{r}\right) u^{\varepsilon}$ is compact in $L^{2}$, then for $t>1$ the family $\nabla_{t, x} u^{\varepsilon}$ is compact in $L^{2}\left(\mathbb{R}^{d}\right)$. The noncompactness has been absorbed at the focus.

(5) A similar phenomenon was described in [RR3] for families $u^{\varepsilon}$ of uniformly dissipative first-order systems when $d=1$ and the intial data are the regularizations $j_{\varepsilon} * \mu$ of finite measures. The $L^{1}(\mathbb{R})$ norm of $u^{\varepsilon}(t)$ decreases in time. It is proved that, for $t>0$, the solutions converge to the solution with initial data given by the nonsingular parts (in the sense of the Lebesgue decomposition) of the measures $\mu_{j}$. The singular part is absorbed. In particular, if the singular part is nonzero then $u^{\varepsilon}(t, x)$ is compact in $L^{1}\left(\mathbb{R}_{x}\right)$ for $t>0$ even though the initial data are not.

(6) The explicit example of Section 5 shows that the result of the Main Theorem is sharp when $h=2$ and $d=4$.

(7) These results were first described in [JMR4].

\section{Analysis of the Singularities}

The most important step in the proof of the Main Theorem is to analyze the jump discontinuities in the derivatives of the solution for times $0 \leq t \leq 1$. The singularities come from the initial jump discontinuities on the sphere $|x|=1$.

The finite speed of propagation implies that the solution $u$ in the Main Theorem satisfies $u=0$ in the truncated cone $|x|<1-t$. Uniqueness implies that $u$ is radial. With the usual abuse of notation we write $u=u(t, r)$, and the differential equation in $\{r>0\}$ becomes

$$
u_{t t}-u_{r r}-\frac{d-1}{r} u_{r}+F_{h}\left(u_{t}\right)=0 .
$$

This is a hyperbolic equation, and the coefficient $(d-1) / r$ is smooth in $\{r>0\}$. The solution we are looking at vanishes in $\{r<1-t\}$ and so is supported in the smooth coefficient region for $0 \leq t<1$.

\section{LEMMA 2.1. (See Figure 3.)}

(i) (Piecewise Continuity for $t<1$ ) If Assumption 1.3 is satisfied then, for $0 \leq$ $t<1, u$ is continuous and piecewise $C^{2}$ with jumps in the first derivatives restricted to the cones $\{|x|=1 \pm t\}$.

(ii) (Piecewise Continuity up to $t=1$ away from $x=0$ ) For any $\delta>0$, u is continous and piecewise $C^{2}$ in the regions $\{r \geq \delta+t, 0 \leq t \leq 1\}$, with jumps in the first derivatives restricted to the cones $\{|x|=1 \pm t\}$. 


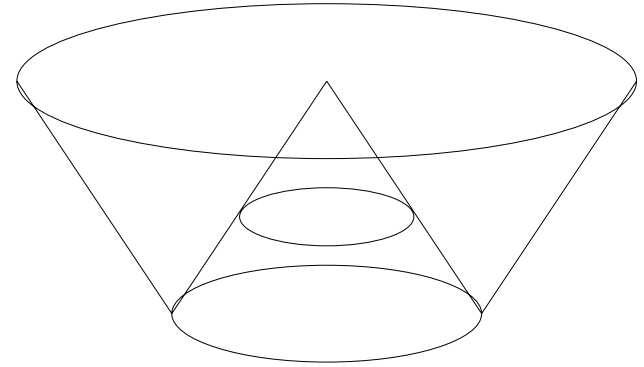

Figure 3 The singularity locus for $0<t<1$

Proof. (i) Fix $0<T<1$. Finite speed shows that, for $0 \leq t \leq T$, $u$ is supported in $r \geq 1-T>0$, where the coefficient $(d-1) / 2 r$ in (2.1) is smooth.

The first step is to show that the solution is uniformly Lipshitzean on $[0, T] \times \mathbb{R}^{d}$. Write (2.1) in the characteristic form

$$
\left(\partial_{t} \pm \partial_{r}\right) u_{ \pm}+\frac{d-1}{2 r}\left(u_{+}-u_{-}\right)+\frac{F_{h}\left(u_{+}+u_{-}\right)}{2^{h}}=0, \quad u_{ \pm}:=\left(\partial_{t} \mp \partial_{r}\right) u .
$$

The standard local existence theorem for hyperbolic equations in space dimension $d=1$ shows that $u$ is uniformly Lipshitzean on $\left[0, T_{1}\right] \times\left[1-T, \infty\right.$ [ with $T_{1}$ small positive. The same result shows that, in order to prove that $u$ is Lipshitzean up to time $T$, it suffices to prove an a priori estimate for $\left\|\nabla_{t, r} u(t)\right\|_{L^{\infty}(\mathbb{R})}$. Precisely, it suffices to show that there is an $M<\infty$ depending only on $f, g$, so that, if $0 \leq t \leq T_{2} \leq T$ and $u$ is a Lipshitzean solution on $\left[0, T_{2}\right] \times[1-T, \infty[$, then $\left\|\nabla_{t, r} u\right\|_{L^{\infty}\left(\left[0, T_{2}\right] \times[1-T, \infty[) \leq M\right.} \leq$.

Multiply (2.2) by $p u_{ \pm}^{p-1}$ with even integer $p$ and add the resulting identities to find that

$$
\begin{aligned}
\partial_{t}\left(u_{+}^{p}+u_{-}^{p}\right)+\partial_{r}\left(u_{+}^{p}-u_{-}^{p}\right)+ & \frac{p(d-1)}{2 r}\left(u_{-}-u_{+}\right)\left(u_{+}^{p-1}+u_{-}^{p-1}\right) \\
& =-\frac{p}{2^{h}}\left(u_{+}^{p-1}+u_{-}^{p-1}\right) F_{h}\left(u_{+}+u_{-}\right) \leq 0 .
\end{aligned}
$$

Define

$$
\psi(t, p):=\int_{1-T}^{\infty} u_{+}^{p}+u_{-}^{p} d r .
$$

Integrate $(2.3)$ over $[0, t] \times[1-T, \infty]$. Integrating by parts and using the fact that $r \geq 1-T>0$, so no boundary terms arise, yields

$$
\begin{aligned}
\psi(t)-\psi(0) & \leq \int_{0}^{t} \int_{1-T}^{\infty} \frac{p(1-d)}{2 r}\left(u_{+}-u_{-}\right)\left(u_{+}^{p-1}+u_{-}^{p-1}\right) d r d t \\
& \leq c p \int_{0}^{t} \psi(t) d t
\end{aligned}
$$

where 
$c=c(d, T):=\frac{d-1}{1-T} \max _{p \in \mathbb{N}_{\text {even }}} \max _{\left\{u_{+}, u_{-}\right\} \in \mathbb{R}^{2} \backslash 0} \frac{\left(u_{-}-u_{+}\right)\left(u_{+}^{p-1}+u_{-}^{p-1}\right)}{u_{+}^{p}+u_{-}^{p}}<\infty$.

Therefore,

$$
\psi(t, p) \leq \psi(0, p)+c(d, T) p \int_{0}^{t} \psi(t, p) d t,
$$

and Gronwall's inequality yields

$$
\psi(t, p) \leq \psi(0, p) e^{c p t}
$$

with $c$ independent of $p$. Taking the $p$ th root gives a bound on the $L^{p}$ norm of $u_{ \pm}$that is independent of $t \leq T_{2}$ and $p$. Passing to the limit $p \rightarrow \infty$ bounds $\left\|u_{ \pm}(t)\right\|_{L^{\infty}\left(\left[0, T_{2}\right] \times[0, \infty[)\right.} \leq M(f, g)$. This estimate completes the proof that $u$ is a uniformly Lipshitzean solution of (1.1) on [0,T] supported in $\{r \geq 1-t\}$.

When $F_{h}$ is a smooth function (i.e., when $h \in \mathbb{Z}_{\text {odd }}$ ), Theorem 1 of [RR1] applied to the first-order system (2.2) implies that $u$ is piecewise smooth with singularities restricted to the two cones.

If one is interested in showing only that Lipshitz continuous solutions are piecewise $C^{2}$, then the argument of [RR2] requires only that the nonlinear function $F$ be $C^{1}$, which it is in our problem. More generally, if $F \in C^{k}$ then the argument of [RR] can be carried out to study discontinuities in derivatives of order $k+1$. The details for completeing this part of the proof of part (i) are left to the reader.

The proof of (ii) is similar. It suffices to prove an a priori estimate

$$
\left\|\nabla_{t, r} u\right\|_{L^{\infty}\left(\left[0, T_{2}\right] \times\{r \geq \delta+t\}\right)} \leq M(f, g)
$$

with $M$ independent of $T_{2} \leq 1$. Introduce

$$
\Psi(t, p):=\int_{\delta+t}^{\infty} u_{+}^{p}+u_{-}^{p} d r
$$

Integrating (2.3) over the region $\{r \geq \delta+t\} \cap\{0 \leq t \leq \underline{t}\}$ yields

$$
\begin{aligned}
& \Psi(\underline{t}, p)-\Psi(0, p)+\left.2 \int_{0}^{\underline{t}} u_{-}^{p}\right|_{r=\delta+t} d t \\
& \leq \int_{0}^{\underline{t}} \int_{\delta+t}^{\infty} \frac{p(1-d)}{2 r}\left(u_{+}-u_{-}\right)\left(u_{+}^{p-1}+u_{-}^{p-1}\right) d r d t \\
& \leq c(d, \delta) p \int_{0}^{\underline{t}} \Psi(t, p) d t .
\end{aligned}
$$

There is now a boundary term on $r=\delta+t$ that is nonnegative and so improves the estimate. As before, this yields an estimate

$$
\Psi(t, p) \leq \Psi(0, p) e^{c(d, \delta) p t}
$$

with $c$ independent of $p$. Taking the $p$ th root and then the limit $p \rightarrow \infty$ yields the desired Lipshitz estimate. 
The next three lemmas prepare for the application of an energy estimate. That estimate is applied to $w:=\partial u$, and one needs to control the growth of the boundary values of $\left(\partial_{t}-\partial_{r}\right) \partial u$ on the shrinking sphere $r=1-t$ as $t$ increases to 1 . In order to do this, we take advantage of the piecewise smoothness.

The main estimate (2.10) of the next lemma is very important. If the problem had been linear, one would have found that the energy density $r^{(d-1)}\left(u_{-}^{2}+u_{+}^{2}\right)$ was constant on the incoming characteristic. If $(d-1)(h-1)>2$ then the energy density tends to zero as $r \rightarrow 0$, which is a result of the nonlinear dissipative mechanism. For linear dissipation, the energy density would converge to a strictly positive quantity.

In comparing the conditions of this lemma with those of the Main Theorem, it is useful to keep in mind the relation

$$
\frac{2 h}{h-1}-1=\frac{h+1}{h-1} \text {. }
$$

LEMMA 2.2 (Analysis of the Incoming Jump). On the incoming characteristic $r=1-t$, one has $u_{+}=0$. If $d>(h+1) /(h-1)$ then, as $t$ increases to 1 ,

$$
\left|u_{-}(t, 1-t)\right|=\frac{c}{r^{1 /(h-1)}}(1+o(1)),
$$

where the values of $u_{-}$are the limits from above (i.e., from $t>1-r$ ) and the constant $c=c(d, h)$ is given in (2.13). In addition, the tangential derivative satisfies

$$
\begin{gathered}
\left|\left(\partial_{t}-\partial_{r}\right) u_{-}(t, 1-t)\right|=\frac{C}{r^{h /(h-1)}}(1+o(1)), \\
C=C(d, h)=\frac{c(d, h)(d-1)}{2}-\frac{c(d, h)^{h}}{2^{h}} .
\end{gathered}
$$

Proof. By finite speed of propagation, both $u_{+}$and $u_{-}$vanish in $r<1-t$. Also, by the plus equation in (2.2), $u_{+}$is continuous across $r=1-t$, which proves that $u_{+}$vanishes on the incoming characteristic.

Next estimate the boundary values $b$ of $u_{-}$:

$$
b(t, 1-t):=\lim _{\delta \rightarrow 0+} u_{-}(t, 1-t+\delta) .
$$

Note that $b$ is defined only on the characteristic line $\{r=1-t\}$.

Since $u_{-}=0$ below the characteristic, the jump in $u_{-}$from under to over the characteristic is equal to $b$. Since $u_{-}=0$ below $r=1-t$ and $u_{+}$vanishes on both sides, the minus equation of (2.2) reads

$$
\left(\partial_{t}-\partial_{r}\right) b-\frac{d-1}{2 r} b+\frac{F_{h}(b)}{2^{h}}=0 .
$$

Let $\gamma:=r^{(d-1) / 2} b=(1-t)^{(d-1) / 2} b(t, 1-t)$. Then

$$
\begin{aligned}
\left(\partial_{t}-\partial_{r}\right) \gamma & =r^{(d-1) / 2}\left(\left(\partial_{t}-\partial_{r}\right) b-\frac{d-1}{2 r} b\right) \\
& =-r^{(d-1) / 2} \frac{F_{h}(g)}{2^{h}}=\frac{-F_{h}(\gamma)}{2^{h} r^{(d-1)(h-1) / 2}} .
\end{aligned}
$$


Then, since $(d-1)(h-1) / 2 \neq 1$,

$$
\begin{aligned}
\left(\partial_{t}-\partial_{r}\right) \frac{|\gamma|^{-h+1}}{-h+1} & =\frac{\left(\partial_{t}-\partial_{r}\right) \gamma}{F_{h}(\gamma)}=\frac{-1}{2^{h} r^{(d-1)(h-1) / 2}} \\
& =\left(\partial_{t}-\partial_{r}\right) \frac{r^{1-(d-1)(h-1) / 2}}{2^{h}(1-(d-1)(h-1) / 2)} .
\end{aligned}
$$

Thus, along $r=1-t$, the quantity

$$
\frac{1}{(h-1)|\gamma|^{h-1}}-\frac{1}{2^{h-1}((d-1)(h-1)-2) r^{(d-1)(h-1) / 2-1}}
$$

is constant.

The hypothesis $(d-1)(h-1)>2$ guarantees that the exponent of $r$ in the second term is positive; hence, as $t$ increases to 1 , the radius $r$ shrinks to 0 and the second term grows without bound. To compensate for this, the first term tends to infinity. Thus,

$$
\begin{gathered}
|\gamma|=c r^{(d-1) / 2-1 /(h-1)}(1+o(1)), \\
c=c(d, h):=2\left(\frac{(d-1)(h-1)-2}{h-1}\right)^{1 /(h-1)} .
\end{gathered}
$$

In terms of the original variable $b$, the estimate (2.13) is equivalent to (2.10).

To prove (2.11), insert the estimate (2.10) into the identity (2.12).

The energy density along the characteristic is therefore

$$
r^{d-1}\left|u_{-}\right|^{2}=O\left(r^{d-1} r^{-2 /(h-1)}\right)=O\left(r^{d-1-2 /(h-1)}\right),
$$

which is $o(1)$ precisely when the hypothesis of Lemma 2.2 is satisfied.

LEMMA 2.3. If $d$ satisfies the condition $d>(h+1) /(h-1)$ from Lemma 2.2 and if $\alpha$ satisfies $\alpha+d>2 h /(h-1)$, then for each $\partial \in\left\{\partial / \partial_{t}, \partial / \partial x_{1}, \ldots, \partial / \partial x_{d}\right\}$ the limits of the derivatives $\left(\partial_{t}-\partial_{r}\right) \partial u$ from above the incoming light cone satisfy

$$
\int_{\substack{|x|=1-t \\ 0<t<1}} r^{\alpha}\left(\left|\left(\partial_{t}-\partial_{r}\right) \partial u\right|^{2}+\left|\nabla_{\omega} \partial u\right|^{2}\right) d \sigma<\infty,
$$

where $\left|\nabla_{\omega} w\right|$ is the length of the angular derivative, given by $\left|\nabla_{\omega} w\right|^{2}:=$ $\left|\nabla_{x} w\right|^{2}-\left|\partial_{r} w\right|^{2}$, and $d \sigma$ is the element of surface area.

Proof. In $|x| \geq 1-t$, write

$$
\frac{\partial u}{\partial x_{j}}=\frac{\partial u}{\partial r} \frac{\partial r}{\partial x_{j}}=\frac{x_{j}}{r} \frac{\partial u}{\partial r} .
$$

Since $\partial_{r} u$ is spherically symmetric, its angular gradient vanishes and so

$$
\nabla_{\omega} \frac{\partial u}{\partial x_{j}}=\left(\nabla_{\omega} \frac{x_{j}}{r}\right) \frac{\partial u}{\partial r} .
$$

Since $2 \partial_{r} u=u_{-}-u_{+}$and since the second summand vanishes on the incoming light cone, it follows that on $|x|=1-t$ we have 


$$
\left(\nabla_{\omega} \frac{x_{j}}{r}\right) \frac{u_{-}}{2}=\left(\nabla_{\omega} \frac{x_{j}}{r}\right) \frac{u_{-}-u_{+}}{2}=O\left(r^{-1} r^{-1 /(h-1)}\right)=O\left(r^{-h /(h-1)}\right) .
$$

Similarly, by the product rule,

$$
\begin{aligned}
\left(\partial_{t}-\partial_{r}\right) \frac{\partial u}{\partial x_{j}} & =\left(\left(\partial_{t}-\partial_{r}\right) \frac{x_{j}}{r}\right) \frac{\partial u}{\partial r}+\frac{x_{j}}{r}\left(\partial_{t}-\partial_{r}\right) \frac{\partial u}{\partial r} \\
& =\left(\left(\partial_{t}-\partial_{r}\right) \frac{x_{j}}{2 r}\right) u_{-}+\frac{x_{j}}{2 r}\left(\partial_{t}-\partial_{r}\right) u_{-} .
\end{aligned}
$$

Equations (2.10) and (2.11) show that each summand is $O\left(r^{-h /(h-1)}\right)$.

Thus,

$$
\int_{\substack{|x|=1-t \\ 0<t<1}} r^{\alpha}\left(\left|\left(\partial_{t}-\partial_{r}\right) \frac{\partial u}{\partial x_{j}}\right|^{2}+\left|\nabla_{\omega} \partial u\right|^{2}\right) d \sigma \leq C \int_{0}^{1} r^{\alpha} \frac{1}{r^{2 h /(h-1)}} r^{d-1} d r .
$$

This integral is finite if and only if $\alpha-2 h /(h-1)+d-1>-1$, recovering the condition in the lemma.

The remaining derivative $\left(\partial_{t}-\partial_{r}\right) \partial_{t} u=\left(\partial_{t}-\partial_{r}\right) \partial_{r} u$ on the incoming cone, so the square integrability follows from the previous estimates.

LEMMA 2.4 (Analysis of the Outgoing Jump). If Assumption 1.4 is satisfied then $u$ is continuously differentiable on a neighborhood of the outgoing cone $\{|x|=$ $1+t, t>0\}$. In particular, $u$ is locally $\mathrm{H}^{2}$ on the complement of the incoming light cone, $u \in H_{\mathrm{loc}}^{2}\left((] 0,1\left[\times \mathbb{R}^{d}\right) \backslash\{|x|=1-t\}\right)$.

Proof. From Lemma 2.1(ii), $u$ is continuous; on a neighborhood of $\{r=1+t$, $t>0\}, u$ is piecewise smooth with singularities on $r=1+t$. It is sufficient to prove that the $u_{ \pm}$are continuous across $\{r=1+t, t>0\}$. For $u_{-}$, this follows from equation (2.2) - and the fact that the $u_{ \pm}$are locally bounded.

Next we show that the continuity of $u_{+}(0, r)$ at $r=1$ from Assumption 1.4 implies the continuity of $u_{+}$across the outgoing characteristic $r=1+t$. The jump in $u_{+}$is defined as

$$
\begin{gathered}
{\left[u_{+}\right](t, 1+t):=u_{+}(t+, 1+t)-u_{+}(t-, 1+t),} \\
u_{+}(t \pm, 1+t):=\lim _{\delta \rightarrow 0+} u(t \pm \delta, 1+t) .
\end{gathered}
$$

The first step is to show that the $\lim _{t \rightarrow 0}\left[u_{+}\right](t, 1+t)=0$.

Equation $(2.2)_{+}$shows that $\left(\partial_{t}+\partial_{r}\right) u_{+}$is locally bounded. Thus, with $0<\delta<$ $t \ll 1$, integrating this equation shows that

$u_{+}(t+\delta, 1+t)-u(0,1-\delta)=O(t), \quad u_{+}(t-\delta, 1+t)-u(0,1+\delta)=O(t)$.

Letting $\delta \rightarrow 0$ and subtracting shows that

$$
\left[u_{+}\right](t, 1+t)=O(t)+\lim _{\delta \rightarrow 0}\left(u_{+}(0,1-\delta)-u_{+}(0,1+\delta)\right) .
$$

The limit on the right is equal to zero, thanks to Assumption 1.4, and therefore

$$
\lim _{t \rightarrow 0+}\left[u_{+}(t, 1+t)\right]=0 .
$$


Define a smooth function $k(t)$ by

$$
k(t):=u_{+}(t-0,1+t)+u_{-}(t, 1+t)=2 u_{t}(t-0,1+t) .
$$

The transport equation satisfied by the jump $\left[u_{+}\right]$along the outgoing characteristic is derived by taking the difference between the equation $(2.2)_{+}$on the upper and lower sides of the characteristic to find

$$
\left(\partial_{t}+\partial_{r}\right)\left[u_{+}\right]+\frac{d-1}{r}\left[u_{+}\right]+\frac{F_{h}\left(\left[u_{+}\right]+k(t)\right)-F_{h}(k(t))}{2^{h}}=0 .
$$

Now define a $C^{1}$ function

$$
G_{h}(t, s):=\frac{d-1}{r} s+\frac{F_{h}(s+k(t))-F_{h}(k(t))}{2^{h}} \quad \text { with } G_{h}(t, 0)=0
$$

to find the nonlinear transport equation

$$
\left(\partial_{t}+\partial_{r}\right)\left[u_{+}\right]+G_{h}\left(t,\left[u_{+}\right]\right)=0 .
$$

The initial value problem defined by (2.15) and (2.16) has the unique solution $\left[u_{+}\right]=0$, which proves the desired continuous differentiability.

\section{Proof of Part (i) of the Main Theorem}

The next step in the proof is an energy estimate, which begins with the energy identity

$$
w_{t} \square w=\partial_{t}\left(\frac{w_{t}^{2}}{2}+\frac{\left|\nabla_{x} w\right|^{2}}{2}\right)-\sum_{j=1}^{d} \partial_{j}\left(w_{t} \partial_{j} w\right):=\partial_{t} e(t, x)-\sum_{j=1}^{d} \partial_{j}\left(w_{t} \partial_{j} w\right) .
$$

Lemma 3.1 (Energy Estimate). For $0<T<1$, define $\Omega_{T}:=\{(t, x): 1+t>$ $|x|>1-t, 0<t<T\}$ and suppose that $w \in C^{1}\left(\overline{\Omega_{T}}\right), \square w \in L^{1}\left(\Omega_{T}\right)$, and $w_{t} \square w \leq 0$ in $\Omega_{T}$. Define $2 e(w, t, x):=w_{t}^{2}+\left|\nabla_{x} w\right|^{2}$. Then

$$
\begin{aligned}
\int_{1+T>|x|>1-T} e(w, T, x) d x \leq & \int_{\substack{|x|=1-t \\
0<t<T}}\left(\left(w_{t}-w_{r}\right)^{2}+\left|\nabla_{\omega} w\right|^{2}\right) \frac{d \sigma}{2 \sqrt{2}} \\
& +\int_{\substack{|x|=1+t \\
0<t<T}}\left(\left(w_{t}+w_{r}\right)^{2}+\left|\nabla_{\omega} w\right|^{2}\right) \frac{d \sigma}{2 \sqrt{2}} .
\end{aligned}
$$

Proof. Choose a smooth radial function $j(t, r)$ compactly supported in $\Omega_{T}$, and define the mollified functions

$$
w^{\varepsilon}(t, x):=\int w(x+\varepsilon z) j(z) d z .
$$

Integrate (3.1) applied to $w^{\varepsilon}$ over $\Omega_{T-\delta}$ and then integrate by parts to find 


$$
\begin{aligned}
\left.\int_{1+t>|x|>1-t} e^{\varepsilon}(t, x) d x\right|_{t=0} ^{t=T-\delta}= & \int_{\Omega_{T-\delta}} w_{t}^{\varepsilon} \square w^{\varepsilon} d x d t \\
& +\int_{\substack{|x|=1-t \\
0<t<T-\delta}}\left(e^{\varepsilon}(t, x)-w_{t}^{\varepsilon}(t, x) w_{r}^{\varepsilon}(t, x)\right) \frac{d \sigma}{\sqrt{2}} \\
& +\int_{\substack{|x|=1+t \\
0<t<T-\delta}}\left(e^{\varepsilon}(t, x)+w_{t}^{\varepsilon}(t, x) w_{r}^{\varepsilon}(t, x)\right) \frac{d \sigma}{\sqrt{2}} .
\end{aligned}
$$

Passing to the limit $\varepsilon \rightarrow 0$ yields

$$
\begin{aligned}
\left.\int_{1+t>|x|>1-t} e(t, x) d x\right|_{t=0} ^{t=T-\delta}= & \int_{\Omega_{T-\delta}} w_{t} \square w d x d t \\
& +\int_{\substack{|x|=1-t \\
0<t<T-\delta}}\left(e(t, x)-w_{t}(t, x) w_{r}(t, x)\right) \frac{d \sigma}{\sqrt{2}} \\
& +\int_{\substack{|x|=1+t \\
0<t<T-\delta}}\left(e(t, x)+w_{t}(t, x) w_{r}(t, x)\right) \frac{d \sigma}{\sqrt{2}} .
\end{aligned}
$$

Note that the integral over $\Omega_{T-\delta}$ is nonnegative and that the contribution to the left-hand side from $t=0$ vanishes; this yields

$$
\begin{aligned}
\int_{1+T-\delta>|x|>1-T+\delta} e(T-\delta, x) d x \leq & \int_{\substack{|x|=1-t \\
0<t<T-\delta}}\left(e(t, x)-w_{t}(t, x) w_{r}(t, x)\right) \frac{d \sigma}{\sqrt{2}} \\
& +\int_{\substack{|x|=1+t \\
0<t<T-\delta}}\left(e(t, x)+w_{t}(t, x) w_{r}(t, x)\right) \frac{d \sigma}{\sqrt{2}} .
\end{aligned}
$$

Simplifying the boundary terms by using the identities

$$
2\left(e \mp w_{t} w_{r}\right)=\left(w_{t} \mp w_{r}\right)^{2}+\left|\nabla_{\omega} w\right|^{2}
$$

and passing to the limit $\delta \rightarrow 0$ proves the lemma.

End of Proof of Main Theorem 1.5(i). For $\partial \in\left\{\partial_{t}, \partial / \partial x_{1}, \ldots, \partial / \partial x_{d}\right\}$, let $w:=$ $\partial u$. Lemma $2.1(\mathrm{i})$ proves that $w \in C^{1}\left(\overline{\Omega_{T}}\right)$ for any $T<1$. Applying $\partial$ to equation (1.1) shows that $\square w=-F_{h}^{\prime}\left(w_{t}\right) w_{t}$, so $w_{t} \square w=-F_{h}^{\prime}(w)\left(w_{t}\right)^{2} \leq 0$ because of the monotonicity of $F_{h}$. Thus Lemma 3.1 can be applied to this $w$.

Next consider the terms on the right-hand side of (3.2) in the limit $T \rightarrow 1$. Lemma 2.1(ii) implies that the second term is bounded independently of $T$. Similarly, Lemma 2.3 with $\alpha=0$ shows that the integral of the first summand in the first integral on the right of (3.2) is bounded independently of $T$. This is where the hypothesis $d>2 h /(h-1)$ is used.

Taking the limit $T \rightarrow 1$ in (3.2) implies that

$$
\limsup _{T \rightarrow 1} \int_{1+T>|x|>1-T} e(w, T, x) d x<\infty .
$$

Inserting the definition $w=\partial u$, this reads 


$$
\limsup _{T \rightarrow 1} \int_{1+T>|x|>1-T}\left(\partial_{t} \partial u(T, x)\right)^{2}+\left|\nabla_{x} \partial u(T, x)\right|^{2} d x<\infty .
$$

Lemma 2.1(ii), together with the continuous differentiability from Lemma 2.4, implies that

$$
\limsup _{T \rightarrow 1} \int_{|x|>\delta+T}\left(\partial_{t} \partial u(T, x)\right)^{2}+\left|\nabla_{x} \partial u(T, x)\right|^{2} d x<\infty .
$$

Combining the last two estimates shows that

$$
\limsup _{T \rightarrow 1} \int_{|x|>1-T}\left(\partial_{t} \partial u(T, x)\right)^{2}+\left|\nabla_{x} \partial u(T, x)\right|^{2} d x<\infty .
$$

Corollary 1.2 and (1.7) together imply that

$$
\begin{gathered}
\partial u(t, x) \in C\left(\left[0, \infty\left[; H^{1 / 2-\varepsilon}\left(\mathbb{R}^{d}\right)\right),\right.\right. \\
\partial_{t} \partial u(t, x) \in C\left(\left[0, \infty\left[; H^{-1 / 2-\varepsilon}\left(\mathbb{R}^{d}\right)\right) .\right.\right.
\end{gathered}
$$

Estimate (3.3), together with the continuity (3.4), implies that the restriction of $\partial_{t, x} \partial u(1, x)$ to $\left\{\mathbb{R}^{d} \backslash 0\right\}$ is a square integrable function; that is,

$$
\int_{\mathbb{R}^{d} \backslash 0}\left(\partial_{t} \partial u(1, x)\right)^{2}+\left|\nabla_{x} \partial u(1, x)\right|^{2} d x<\infty .
$$

Define $G(x)$ to be the square integrable function that is the restriction of $\partial_{t, x} \partial u(1, x)$ to $\mathbb{R}^{d} \backslash 0$, and let

$$
R(x):=G(x)-\partial_{t, x} \partial u(1, x), \quad \text { so } \quad \operatorname{supp} R \subset\{0\} .
$$

The regularity (3.4) implies that

$$
R \in H^{-1 / 2-\varepsilon}\left(\mathbb{R}^{d}\right) .
$$

Since there are no nonzero elements of this space with support at the origin, it follows that $R=0$ and hence that $\partial_{t, x} \partial u(1, x) \in L^{2}\left(\mathbb{R}^{d}\right)$. Corollary 1.2 with $\sigma=1$ implies that (1.8) is satisfied, so the proof of Main Theorem 1.5(i) is complete.

\section{Proof of Part (ii) of the Main Theorem}

The difference in the analysis comes from the square integrability near the focus at $t=1, r=0$. For the second part of the Main Theorem, one needs the weights $r^{\alpha}$ from Lemma 2.3. In order to take advantage of the weighted estimates from Lemma 2.3, we use the following weighted energy estimate, which reduces to Lemma 3.1 when $\alpha=0$.

Lemma 4.1 (Weighted Energy Estimate). For $0<T<1$, define $\Omega_{T}:=\{(t, x)$ : $1+t>|x|>1-t, 0<t<T\}$, and suppose that $w \in C^{1}\left(\overline{\Omega_{T}}\right)$ and $0 \leq w_{t} \square w \in$ $L^{1}\left(\Omega_{T}\right)$. Define $2 e(t, x):=w_{t}^{2}+\left|\nabla_{x} w\right|^{2}$. Then, for all $\alpha \geq 0$, 


$$
\begin{aligned}
& \int_{1+T>|x|>1-T}(|x|+1-T)^{\alpha} e(T, x) d x \\
& \leq \int_{\substack{|x|=1-t \\
0<t<T}}(|x|+1-t)^{\alpha}\left(\left(w_{t}-w_{r}\right)^{2}+\left|\nabla_{\omega} w\right|^{2}\right) \frac{d \sigma}{2 \sqrt{2}} \\
& \quad+\int_{\substack{|x|=1+t \\
0<t<T}}(|x|+1-t)^{\alpha}\left(\left(w_{t}+w_{r}\right)^{2}+\left|\nabla_{\omega} w\right|^{2}\right) \frac{d \sigma}{2 \sqrt{2}} .
\end{aligned}
$$

Proof. We give a proof assuming that $w \in C^{2}\left(\overline{\Omega_{T}}\right)$. A regularization as in the proof of Lemma 3.1 shows that this is sufficient.

Multiplying (3.1) by a continuous function $\phi(t, x)$ with integrable first derivatives yields

$$
\begin{aligned}
\partial_{t}(\phi e(t, x))-\sum_{j=1}^{d} \partial_{j}\left(\phi w_{t} \partial_{j} w\right) & =\phi_{t} e-\sum_{j=1}^{d}\left(\partial_{j} \phi\right)\left(w_{t} \partial_{j} w\right) \\
& \leq \phi_{t} e+\left|\nabla_{x} \phi\right|\left|w_{t}\right|\left|\nabla_{x} w\right| \leq\left(\phi_{t}+\left|\nabla_{x} \phi\right|\right) e .
\end{aligned}
$$

If $\phi$ satisfies $\phi_{t}+\left|\nabla_{x} \phi\right| \leq 0$, then an integration by parts in $\Omega_{T}$ yields

$$
\begin{aligned}
0 \geq & \left.\int_{1+t>|x| \geq 1-t} \phi(t, x) e(t, x) d x\right|_{t=0} ^{t=T} \\
& -\int_{\substack{|x|=1-t \\
0<t<T}} \phi(t, x)\left(e(t, x)-w_{t}(t, x) w_{r}(t, x)\right) \frac{d \sigma}{\sqrt{2}} \\
& -\int_{\substack{|x|=1+t \\
0<t<T}} \phi(t, x)\left(e(t, x)+w_{t}(t, x) w_{r}(t, x)\right) \frac{d \sigma}{\sqrt{2}} .
\end{aligned}
$$

Taking $\phi(t, x):=(|x|+1-t)^{\alpha} \geq 0$ and using the identities $2\left(e \mp w_{t} w_{r}\right)=$ $\left(w_{t} \mp w_{r}\right)^{2}+\left|\nabla_{\omega} w\right|^{2}$ yields (4.1).

Proof of Main Theorem 1.5(ii). Using Lemma 2.1(ii), Lemma 2.4, and estimates (2.14) and (4.1), and reasoning as in the proof of Theorem 1.5(i), yields the following weighted estimates on $\mathbb{R}^{d} \backslash 0$. Define $\left.\left.\alpha \in\right] 0,1 / 2\right]$ by

$$
\alpha:=\frac{1}{2}\left(\frac{2 h}{h-1}-d\right) \text {. }
$$

Then, for all $\varepsilon>0$ and $\partial \in\left\{\partial / \partial t, \partial / \partial x_{j}\right\}$,

$$
\int_{\mathbb{R}^{d} \backslash 0}|x|^{2 \alpha+2 \varepsilon}\left(\left(\partial_{t} \partial u(1, x)\right)^{2}+\left|\nabla_{x} \partial u(1, x)\right|^{2}\right) d x<\infty .
$$

An application of Hölder's inequality shows that the function defined for $x \neq 0$ by $\partial_{x} \partial u(1, x)$ is absolutely integrable on compact subsets of $\mathbb{R}^{d}$ and so defines a distribution. As in the sentence before equation (3.6), this distribution is called $G(x)$. Define $R$ as in (3.6). Then 


$$
R \in L_{\mathrm{loc}}^{1}\left(\mathbb{R}^{d}\right)+H^{-1 / 2-\varepsilon}\left(\mathbb{R}^{d}\right) \text { and } \operatorname{supp} R \subset\{0\} .
$$

It follows that $R=0$. Thus, $G$ is equal to $\partial_{x} \partial u(1, x)$, where the derivatives are taken in the sense of distributions. Thus,

$$
\Phi:=\partial u \Longrightarrow|x|^{\alpha-\varepsilon} \nabla_{x} \Phi \in L^{2}\left(\mathbb{R}^{d}\right) .
$$

LEMma 4.2 (Hardy Inequality). If $\beta \in] 0, d / 2[$ then there is a $c=c(d, \beta)$ such that, for all $\Phi \in \mathcal{S}\left(\mathbb{R}^{d}\right)$,

$$
\left\||D|^{1-\beta} \Phi\right\|_{L^{2}\left(\mathbb{R}^{d}\right)} \leq c\left\||x|^{\beta} \nabla_{x} \Phi\right\|_{L^{2}\left(\mathbb{R}^{d}\right)} .
$$

Proof. Inequality (4.6) follows from the inequality

$$
\left\||D|^{-\beta} \psi\right\|_{L^{2}\left(\mathbb{R}^{d}\right)} \leq c\left\||x|^{\beta} \psi\right\|_{L^{2}\left(\mathbb{R}^{d}\right)}, \quad 0<\beta<\frac{d}{2},
$$

applied to the first derivatives of $\Phi$. Inequality (4.7) is, in turn, a consequence of the boundedness on $L^{2}$ of the integral operator with kernel

$$
\frac{1}{|x-y|^{d-\beta}} \frac{1}{|y|^{\beta}}, \quad 0<\beta<\frac{d}{2} .
$$

(A proof of this boundedness can be found in [SW].) This completes the proof of Lemma 4.2.

Applying (4.6) to the regularizations $\Phi^{\varepsilon}:=j_{\varepsilon} * \partial u$ and passing to the limit $\varepsilon \rightarrow 0$ yields

$$
|D|^{1-\alpha-\varepsilon} \partial u(1, \cdot) \in L^{2}\left(\mathbb{R}^{d}\right) .
$$

An application of Corollary 1.2 completes the proof of the Main Theorem.

\section{An Explicit Example}

In this section we compute an explicit example that exhibits smoothing of a singularity. The example is self-similar, so the partial differential equation in $t, r$ becomes a nonlinear equation with singularities of Fuchs type. When $h=2$, this equation is explicitly solvable.

If $v$ is a solution of (1.1) and $\lambda>0$, then

$$
u_{\lambda}=u_{\lambda}(t, x):=\lambda^{\alpha} v(\lambda t, \lambda x)
$$

is also a solution, provided that $\alpha$ and $h$ satisfy the equivalent conditions

$$
\alpha=\frac{2-h}{h-1}, \quad h=\frac{\alpha+2}{\alpha+1} .
$$

For the case of quadratic nonlinearity,

$$
h=2 \text { and } \alpha=0,
$$

we seek radial self-similar solutions - that is, solutions satisfying 


$$
u(t, r):=u(\lambda t, \lambda r)
$$

Setting $\lambda=1 / r$ shows that

$$
u(t, r)=u(t / r, 1):=U(t / r) .
$$

Then

$$
\begin{gathered}
u_{t}=\frac{1}{r} U^{\prime}\left(\frac{t}{r}\right), \quad u_{t t}=\frac{1}{r^{2}} U^{\prime \prime}\left(\frac{t}{r}\right), \\
u_{r}=\frac{-t}{r^{2}} U^{\prime}\left(\frac{t}{r}\right), \quad u_{r r}=\frac{t^{2}}{r^{4}} U^{\prime \prime}\left(\frac{t}{r}\right)+\frac{2 t}{r^{3}} U^{\prime}\left(\frac{t}{r}\right) .
\end{gathered}
$$

Therefore, equation (2.1) may be written as

$$
0=\square u+F_{h}\left(u_{t}\right)=\left[\frac{1}{r^{2}}-\frac{t^{2}}{r^{4}}\right] U^{\prime \prime}+\left[\frac{(d-1) t}{r^{3}}-\frac{2 t}{r^{3}}\right] U^{\prime}+\frac{1}{r^{2}} U^{\prime}\left|U^{\prime}\right| .
$$

Multiply by $r^{2}$, and set

$$
s:=t / r \quad \text { and } \quad V:=U^{\prime}
$$

to find

$$
\left(1-s^{2}\right) V^{\prime}+(d-3) s V+V|V|=0 .
$$

Consider solutions with

$$
U=V=0 \text { for }-\infty<s<-1,
$$

which corresponds to solutions $u$ that vanish on the incoming cone $\{t<-r\}$.

For $-1<s<1$, use the change of variable

$$
V:=\left(1-s^{2}\right)^{(d-3) / 2} W
$$

to find that (5.7) is transformed to

$$
\left(1-s^{2}\right) W^{\prime}+\left(1-s^{2}\right)^{(d-3) / 2} W|W|=0 .
$$

Thus, $W$ never changes sign in $\{-1<s<1\}$ and $-W$ is a solution whenever $W$ is a solution.

Separating variables in (5.8) yields the positive solution

$$
W(s)=\frac{1}{F(s)}, \quad \text { where } F(s):=\int_{-1}^{s}\left(1-t^{2}\right)^{(d-5) / 2} d t .
$$

This integral is finite for $d>3$, and approaching $s=-1$ from above yields

$$
F(s)=\frac{\left(1-s^{2}\right)^{(d-3) / 2}}{d-3}(1+o(1)) .
$$

The right-hand limit of $V(s)$ at $s=-1$ is therefore given by

$$
\lim _{s \searrow-1} V(s)=(d-3) .
$$

Hence $U^{\prime}=V$ has a jump discontinuity at $s=-1$, so the first derivative of the self-similar solution has a jump discontinuity on the incoming light cone. 
When $s$ increases to +1 from below, one has

$$
V(s) \sim c\left(1-s^{2}\right)^{(d-3) / 2} .
$$

Extend $V$ to vanishing for $s>1$,

$$
V:=0 \text { for } s \geq 1 \text {. }
$$

The resulting self-similar solution is constant inside the outgoing light cone $\{r=$ $t>0\}$. In addition, the first derivatives of $u$ are continuous across this cone.

Near the outgoing cone,

$$
\nabla_{t, r} u \sim(r-t)^{(d-3) / 2}
$$

and so, for all $\varepsilon>0$,

$$
u \in H_{\mathrm{loc}}^{(1+(d-3) / 2+1 / 2-\varepsilon)} .
$$

For $d=4$, this example shows that the result of the Main Theorem is sharp. For $d>4$, the regularity on the outgoing cone increases linearly with $d$ as if the result of the second part of the Main Theorem were true for all $d>2 h /(h-1)$.

\section{References}

[B] J.-M. Bony, Intéraction des singularités pour les équations aux dérivées partielles nonlinéaires, Sém. Goulaouic-Meyer-Schwartz (Palaiseau, France, 1982).

[CH] R. Courant and D. Hilbert, Methods of mathematical physics, vol. I, Wiley, New York, 1953.

[GR] P. Gerard and J. Rauch, Propagation de la régularité locale de solutions d'équations hyperboliques nonlinéaires, Ann. Inst. Fourier (Grenoble) 37 (1987), 65-85.

[JMR1] J.-L. Joly, G. Métivier, and J. Rauch, Focusing and absorbtion of nonlinear oscillations, Journées aux dérivées partielles (St. Jean de Monts, 1993), vol. III, pp. 1-11, École Polytéchnique, Palaiseau, France.

[JMR2] - Focusing at a point and absorbtion of nonlinear oscillations, Trans. Amer. Math. Soc. 347 (1995), 3921-3969.

[JMR3] - Caustics for dissipative semilinear oscillations, Geometric optics and related topics (F. Colombini, N. Lerner, eds.), pp. 245-266, Birkhäuser, Boston, 1997.

[JMR4] - Nonlinear hyperbolic smoothing at a focal point, Seminaire équations aux derivées partielles (1998-99), École Polytéchnique, Palaiseau, France.

[JMR5] - $-L^{p}$ estimates for oscillatory integrals and caustics for dissipative semilinear oscillations, Mem. Amer. Math. Soc. 144 (2000).

[LS] J.-L. Lions and W. Strauss, Some nonlinear evolution equations, Bull. Soc. Math. France 93 (1965), 43-96.

[RR1] J. Rauch and M. Reed, Jump discontinuities of semilinear, strictly hyperbolic sytems in two variables: Creation and propagation, Comm. Math. Phys. 81 (1981), 203-227.

[RR2] - Striated solutions of semilinear, two-speed wave equations, Indiana Univ. Math. J. 34 (1985), 337-353. 
[RR3] - Nonlinear superposition and absorption of delta waves in one space dimension, J. Funct. Anal. 73 (1987), 152-178.

[RR4] - Bounded stratified and striated solutions of hyperbolic systems, Nonlinear partial differential equations and their applications, vol. IX (H. Brezis, J. L. Lions, eds.), pp. 334-351, Pitman Res. Notes Math. Ser., 181, Longman, Harlow, U.K., 1989.

[SW] E. Stein and G. Weiss, Fractional integrals on $n$-dimensional Euclidean space, J. Math. Mech. 7 (1958), 503-514.

J.-L. Joly

MAB

Université de Bordeaux I

33405 Talence

France

joly@math.u-bordeaux.fr

G. Metivier

IRMAR

Université de Rennes I

35042 Rennes

France

metivier@univ-rennes1.fr

J. Rauch

Department of Mathematics

University of Michigan

Ann Arbor, MI 48109

rauch@math.lsa.umich.edu 